\title{
Rigorous Microscopic Drift-Diffusion Theory and its Applications to Nanostructures
}

\author{
M. Orlowski \\ Advanced Products Research and Development Laboratory, Motorola Inc. \\ 3501 Ed Bluestein Blvd., Austin, TX 78721, USA
}

\begin{abstract}
A recently proposed approach [1] to describe rigorously diffusion and diffusionrelated reactions on arbitrary networks in terms of elementary jumps has been extented to include drift-diffusion capability. The method is based on combined density function approach to diffusion and on an adjacency matrix concept used in graph theory. The new method allows flexible and selective mixing of drift and diffusion on any two-dimensional domain of arbitrary outer and inner geometry. In particular, partial identity rank 4 tensors are introduced to allow preservation of results of previous operations not affected by the subsequent mechanisms. As an application it is shown that switching-off behavior of currents depends sensitively on the domain geometry.
\end{abstract}

\section{Introduction to the Tensorial Diffusion Approach}

The present approach is based on a tensorial formulation of the adjacency matrix in $2 \mathrm{D}$ proposed recently [1] to describe rigorously diffusion on arbitrary grain boundary networks. To calculate elementary jumps in $2 \mathrm{D}$ lattice with nodes $(i, j)$ a four dimensional tensor $\mathcal{T}$ is defined with elements $\tau(i, j, m, n)=1$ if the nodes $(i, j)$ and $(m, n)$ are connected with each other, and $\tau(i, j, m, n)=0$ otherwise. Note that the connection is directional, i.e. the node $(i, j)$ can be connected with the node $(m, n)$ but not vice versa. That means that a particle can jump from $(i, j)$ to $(m, n)$ but not necessarily back. Clearly, this property allows to define drift along arbitrary axis and also any local emission and absorption mechanisms. For example, to describe diffusion allowing jumps to the nearest neighbors, only tensor elements which differ in only one of their coordinates by a unity will have the value one and all other zero: i.e., for example, $\tau(i, j, i+1, j)=1$. To evaluate the consecutive jumps in the entire domain a specific tensor product has to be constructed. This is accomplished by the following definition: $\mathcal{T} \bullet \mathcal{T} \equiv\left(\tau^{(2)}(i, j, m, n)\right)=\sum_{(k, l)} \tau(i, j, k, l) \cdot \tau(k, l, m, n)$. In order to evaluate the M-th tensor product, a tensor contraction $\hat{\mathcal{T}}_{(i, j)}^{(M)}$ with elements $\hat{\tau}_{(i, j)}^{(M)}(m, n)$ is defined. The contracted tensor gives the probability for the particle which started to diffuse at the node $(i, j)$ to arrive after $\mathrm{M}$ jumps at nodes $(m, n) \forall m$ and $n$. To illustrate this, consider the diffusion of an implanted profile $g\left(x_{i}, y_{j} ; t=0\right)$ for a time $t=t^{*} \geq 0$. If $f$ is the frequency of the diffusion coefficient then $M=f \cdot t^{*}$ and and the evolution of the dopant profile is given by $g\left(x_{m}, y_{n} ; t=t^{*}\right)=\sum_{(i, j)} g(i, j ; t=0) \cdot \hat{\tau}_{(i, j)}^{(M)}(m, n)$. 


\section{Extension to Drift and Interactions}

So far, the method [1] has been employed random walk diffusional jumps to the nearest neighboring nodes described by the tensor $\mathcal{T}_{s r}$ which we now characterize by an additional subscript $s r$, for short range. However, in the present formalism other mechanisms can be considered easily, since all mechanisms are defined by a specific distribution of the $1 \mathrm{~s}$ and the 0 s of the respective tensor elements. For example, long range diffusional jumps will be defined by a tensor $\mathcal{T}_{l r}$, a drift of particles in a specific direction by the tensor $\mathcal{T}_{d}$, absorbtion at certain regions by the tensor $\mathcal{T}_{a b}$, and emission from some regions by the tensor $\mathcal{T}_{e m}$, and so on. To consider a composite dynamics of all the mechanisms invoked, we have simply to form a multiple product of the elementary mechanisms considered, followed by a power tensor of this product: $\left(\mathcal{T}_{e m}^{n 5} \bullet \mathcal{T}_{a b}^{n 4} \bullet \mathcal{T}_{d}^{n 3} \bullet \mathcal{T}_{l r}^{n 2} \bullet \mathcal{T}_{s r}^{n 1}\right)^{M}$, where the exponents $n i(i=1, . ., 5)$ denote the relative rapidity of the mechanism compared with the others, and $M$ determines how often the whole cycle is supposed to take place, i.e., properly scaled $M$ describes the process time.

The incorporation of drift is easily accomplished. Suppose the drift is along the $\mathrm{x}$ axis (with the index $i$ ), then the only non-zero tensor elements are $\tau(i, j, m, j)=1$ if $m=i+1$ and $n=j$. Suppose the drift is along the xy-diagonal of the domain then the respective drift is a composite drift of lateral drift, $\tau_{l a t}(i, j, m, n)=1$ only if $m=i$ and $n=j+1$ and zero otherwise, and by a subsequent vertical drift downwards, given by $\tau_{\text {ver }}(i, j, m, n)=1$ only if $m=i+1$ and $n=j$ and zero otherwise. The composite operation describing the drift downward the diagonal is accomplished by applying the tensor product $\mathcal{T}_{\text {drif }}=\mathcal{T}_{\text {ver }} \bullet \mathcal{T}_{\text {lat }}$.

In many cases there might be parts of the domain which are not affected by a specific operation, for example by drift in a selected subdomain $\mathcal{D}_{s}$, or even on the same subdomain there might be mechanisms operating on different time scales. In this case the tensor reflecting the operation on the subdomain, $\mathcal{D}_{s}$, will have zero tensor elements outside this particular subdomain, and by necessity, zero tensor elements representing all other mechanisms. Because of these vanishing tensor elements, the results of all previous operations and existing distributions outside of the subdomain will not be preserved under the current operation. In order to preserve the results of previous operations in subdomains not considerered under current time step, the currently applied tensor has to be partitioned and its elements pertaining to these domains should be defined im the following way: $\tau_{c u r r}(i, j, m, n)=1$ only if $m=i$ and $n=j$. In terms of our earlier definition this amounts to the self-connectedness relation, i.e. the node $(i, j)$ (where $\left(x_{i}, y_{j}\right) \in \mathcal{D}_{r} \forall r \neq s$ ) is connected to itself. This identity operation preserves the dopant distribution obtained so far by previous operations in the respective subdomains.

The present approach allows also a definition of interactions on a two- (and, in principle, also on a three-) dimensional lattice. Suppose the nodes $(m, n)$ and $(r, s)$ are initially not connected with one another, i.e. $\tau(m, n, r, s)=0$ and $\tau(r, s, m, n)=0$. The connection (i.e. interaction) can be activated at a later stage in the evolution, say at $t=t_{o}$ by turning the tensor elements to be, $\tau(m, n, r, s)=1$ and/or $\tau(r, s, m, n)=1$, depending on the kind of interaction considered, as soon as the values of the species density $g\left(i, j, t=t_{o}\right)$ under consideration at the nodes $(m, n)$ and $(r, s)$ are reaching specific values, for example. Construction of these interactions and their evaluation will be subject of future work. 


\section{Application of Drift-Diffusion}

As an illustration of the extended formalism expounded in the preceding section, we consider drift diffusion in two structures, one consisting of simple conducting rectangular strip with $\mathrm{x}$-axis along the drift direction Fig.1a, and a second structure, in which the latter rectangular domain has been extended in the transversal direction (y) by narrow rectangles, forming a cross structure, as shown in Fig.1b. In the initial stage of the drift-diffusion transport, the species, for example, electrons, are entering both structures at $x=0$ and are allowed to spread in the structures by means of diffusion only. A typical diffusion profile for the cross structure is shown in Fig.2.
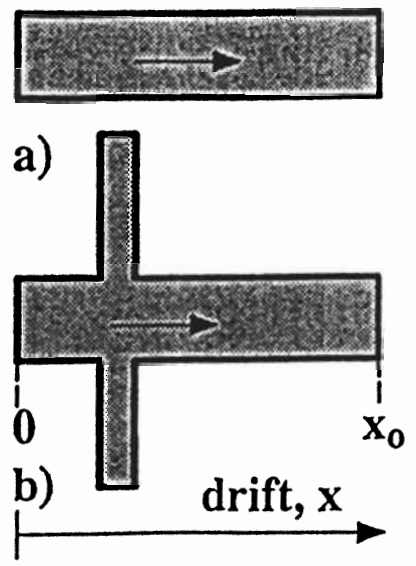

Fig.1: Two structures used for drift diffusion studies: a) rectangular channel and b) rectangular channel with transversal arms (cross structure).

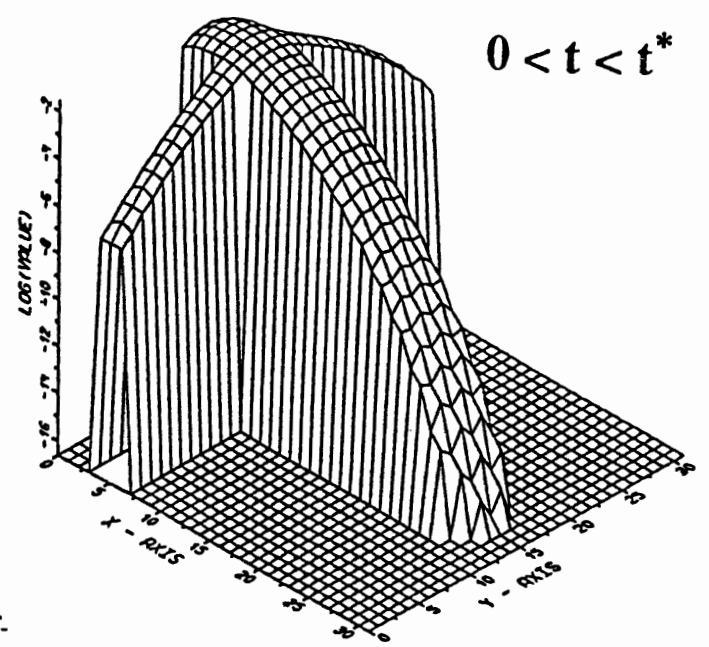

Fig.2: Diffusion profile of the species (electrons) in the cross structure prior to switching on of the drift field.

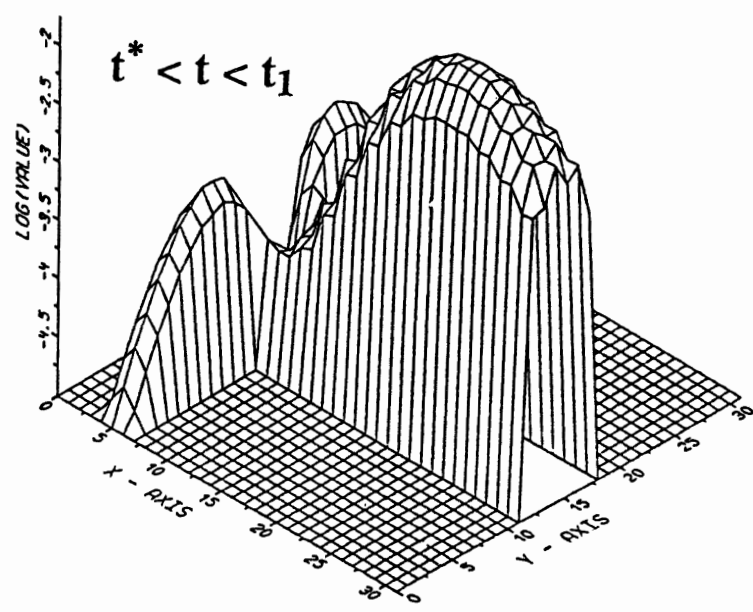

Fig.3: Electron distribution in the cross structure shortly after the drift field has been switched on.
It can be seen that the electrons spread also sideways into the lateral arms of the cross structure. At $t=t^{*}$ the drift (field) along the $\mathrm{x}$-axis is switched on. After some time the diffusion profiles is shifted downwards under the influence of the drift field along the $\mathrm{x}$-axis as shown in Fig.3. Let us now suppose that at a later time, $t=t_{1} \geq t^{*}$, the supply of electrons at $x=0$ has been cut-off. In the case of the simple rectangular structure, it is clear, that cutting-off the supply of electrons will let the current drop abruptly after a delay time $\Delta t=x_{o} / v$, where $x_{o}$ is the length of the rectangular channel and $v$ is the drift velocity. This means that $\Delta t$ is 


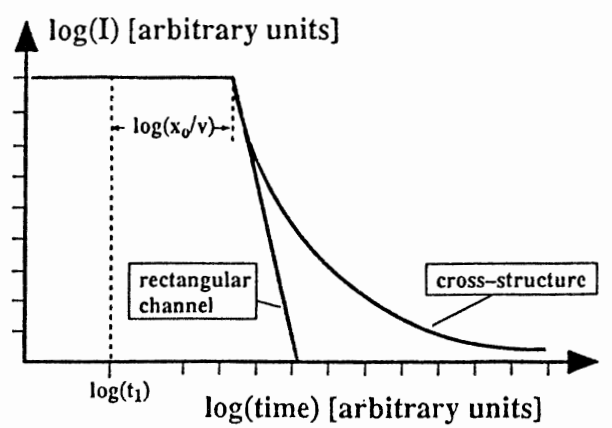

Fig.4: Current switching-off characteristics for rectangular channel and for the cross structure. the time needed to deplete the channel completely. The current switch-off characteristics will exhibit some decaying tail, due to the fact that the cut-off electron packet has been broaden in the rectangular channel by diffusion. This is shown in Fig.4. The switching-off behavior for the cross structure is entirely different. Even at times considerably larger than $t=t_{1}+\Delta t$, there will be still considerable level of current, flowing out of the channel, which is fed by the electrons outdiffusing from the lateral cross arms into the main rectangular channel. Once in the rectangular channel the electrons are subject not only to diffusion but also to drift along

the $\mathrm{x}$-axis, which, eventually, depletes them from the channel. This case is shown in Fig.5. The electron distributions for all times sufficiently larger than $t=t_{1}+\Delta t$ are self-similar. This means that for any time $t \gg t_{1}+\Delta t$ the electron distributions looks exactly the same way, except, of course, as should be expected, for the absolute concentration level, which decreases as the time goes on. It lies in the nature of diffusion (entropy) that it takes finite time for the electrons to diffuse into the regions transversal to the drift flow, but it takes an infinite time to deplete them completely, even having, as in the present case, a 'sink' at the intersection with the drift region. This qualitatively different switching behavior of the current in the cross structure is shown also in Fig.4. This example suggests, that at nanoscale dimensions an appropriate streamlining of the device structure will improve the switching properties of the device significantly.

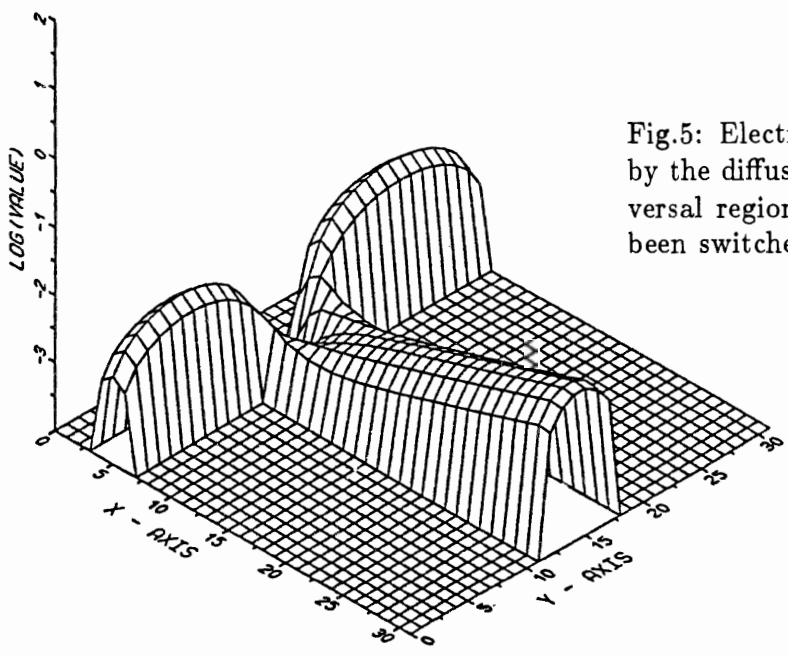

\section{References}

[1] Marius Orlowski, IEDM'92 Proceedings, p.161, San Francisco, 1992 